\title{
Spatiotemporal Distribution of Gastrointestinal Tract Cancer through GIS over 2007-2012 in Kermanshah-Iran
}

\author{
Sohyla Reshadat ${ }^{1}$, Shahram Saeidii ${ }^{2 *}$, Ali Reza Zangeneh ${ }^{2}$, Nahid Khademi ${ }^{3}$, \\ Keyvan Khasi ${ }^{4}$, SayedRamin Ghasemi ${ }^{5}$, Nader Rajabi Gilan ${ }^{6}$
}

\begin{abstract}
Background: Cancer is one of the common causes of disability and mortality in the world. The present study aimed to define the spatiotemporal distribution of gastrointestinal tract cancers using a geographic information system (GIS) over the time period of 2007-2012 in Kermanshah-Iran. Materials and Methods: The method of studying was descriptive-analytical as well as comparative with gastrointestinal tract cancer patients based in the City of Kermanshah over the time period covered. For data analysis, the GIS and SPSS 16.0 were applied. Results: According to the pathological reports within the space of 5 years, 283 cases of gastrointestinal tract cancer (157 in males, 156 in females) were reported. The performed tests in terms of spatial distribution in the environment of GIS indicated that the disease demonstrated a clustered pattern in the City of Kermanshah. More to the point, some loci of this disease have emerged in the City of Kermanshah that in the first level, 6 neighborhoods with 29-59 cases of this disease per square kilometer and in the second level, 15-29 cases. Conclusions: Gastrointestinal tract cancer demonstrated an ascending trend within the space of 5 years of research and the spatiotemporal distribution of cancer featured a concentrated and clustered pattern in the City of Kermanshah.
\end{abstract}

Keywords: Spatiotemporal distribution - cancer - geographic information system - Kermanshah, Iran

Asian Pac J Cancer Prev, 16 (17), 7737-7742

\section{Introduction}

The gastrointestinal tract cancers are one of the most important types of cancer in terms of prevalence and mortality. Stomach cancer is reported to be the fourth common cancer(Sadjadi et al., 2003; Murphy et al., 2009). It is estimated that in the next decades due to the environmental hazards, aging and changing life style cancer will be more common(May, 1950).

Prevalence rate of the gastrointestinal tract cancer in Iran is high especially in the west part of Iran(Sadjadi et al., 2003; RAHIMI and HEIDARI, 2012). The latest data by the Iran National Cancer Registry (INCR) reported an annual number of 51,000 cases of cancer with about 35,000 cancer-related deaths in the country. This is the second highest in the Eastern Mediterranean region according to the World Health Organization reports. The same report shows that CRC was the fourth most common cancer in Iran between 2000 and 2009(Radmard, 2010; Hajmanoochehri et al., 2014). 38\% of cancers reported in Iran are accompanied by the gastrointestinal tract cancer(Fathollahi et al., 2011). It is well recognized that the northern part of Iran to be the region with the highest incidence of the gastrointestinal tract cancer(Somi et al., 2006).

The geographical distribution of the gastrointestinal tract cancer suggests a relationship between its occurrence and surroundings(Wu et al., 2008; Merletti et al., 2011). Locations play an important role in the epidemiology of diseases. Hippocrates illustrated in his memorial book, 'Airs, Waters, and Places' that, you will find, as a general rule, that the constitutions and habits of people follow the nature of the land where they live in. He observed the relationship between the environment and human health. The initial disease maps were produced in Germany over two hundred years ago. Then in 1855, dot maps of cholera epidemic, by John Snow became the most famous example of spatial epidemiology(Snow, 1855). In nineteenth century research looked to geography to identify etiological clues(May, 1950; Krieger, 2000; McLeod, 2000). In the 1970s, using of GIS accumulated among epidemiologists and public health researchers(Clayton and Kaldor, 1987).

Geographic information system (GIS) is useful for

${ }^{1}$ Pediatrician, Center of Excellence for Community Oriented Medicine Education and Social Development \& Health Promotion Research Center, ${ }^{2}$ Urban Planning and Geography, Social Development \& Health Promotion Research Center, ${ }^{3}$ Director of Disease Control of Vice-chancellor of Health, ${ }^{4}$ Health Department Kermanshah University of Medical Sciences, ${ }^{5}$ Social Welfare, Social Development \& Health Promotion Research Center, ${ }^{6}$ Social Research, Social Development \& Health Promotion Research Center, Kermanshah University of Medical Sciences, Kermanshah, Iran *For correspondence: saedishahram@yahoo.com 
identifying The geographic characteristics of residential areas(Morra et al., 2006), preparing information regarding phenomenon related to temporal locations(Rezaeian, 2004), identifying the loci of diseases, and applied for investigating the potential relationship between environmental risk factors and disorders includings: cancer(Openshaw et al., 1987; Roche et al., 2002; Samat and Shattar, 2014) Infectious diseases such as: HIV/AIDS(Mahbubeh et al., 2014; Zulu et al., 2014), multiple sclerosis(Mahbubeh et al., 2014) diabetes(Curtis et al., 2013). GIS can be defined as a computer system with the capacity to capture, store, analyze, and display geographically referenced information, and have been used in a wide variety of fields, including the natural, social, engineering and particularly the medical sciences because of their ability to incorporate physical, biological, cultural, demographic or economic information(Musa et al., 2013).

Medical GIS has become extremely useful in understanding the bigger picture of public health. Health organizations can now visualize, analyze, interpret and display multifaceted geo-location data through the use of GIS tools, mapping applications, and big data(Musa et al., 2013). Epidemiologists use GIS to assess proximity, aggregation, and clustering, as well as to perform spatial smoothing, interpolation and spatial regression. The most common application of GIS in these fields remains the identification of disease clusters, which refer to non random spatial distributions of disease cases, incidence, or prevalence(Auchincloss et al., 2012). GIS has also been used extensively in epidemiology for disease surveillance and intervention monitoring(Clarke et al., 1996). By mapping disease cases in geographic space, local and national governments can easily identify the distribution and spread of disease across geographic regions, optimize planning of inter venation locations, and monitor their effectiveness(Clarke et al., 1996). When the maps of prevalence and spatial distribution of various groups of diseases (e.g. cancers) with unknown causes are produced; some significant differences are observed from one point to the other which would be of considerable assistance to pathological studies and treatment of such diseases. When the loci of diseases are specified, the health and treatment related costs will be moderated because the residents of regions prone to certain diseases understand the risk factors and do their utmost to hamper them(Shad et al.). Identifying the loci of diseases also provides policymakers with new opportunities for estimating the relationship between public health and the geographic characteristics of residential areas(Pearce and Boyle, 2005).

Medical geography has been introduced by Iranian researchers as a need in public health(Shad et al.; Rezaeian, 2004). In Iran most of the existing researches about gastrointestinal tract cancer is on epidemiological studies(Darvishmoghadam et al., 2005; Yazdanbod et al., 2005; Somi et al., 2006; Mehrabani et al., 2008; Radmard, 2010; RAHIMI and HEIDARI, 2012). however a few studies have documented the evaluation of geographical variation of this cancer through GIS. In this regard Tahamtan did his study in Mazandaran and Golestan provinces, tow provinces in the north of
Iran(Mohammadpour Tahamtan et al., 2014), Askarian's study was done in Fars province, Southern Iran(Askarian et al., 2014), and Asmarian did his study in Iran(Asmarian et al., 2013). up to now there is no study have been carried out regarding applying GIS to investigate this subject in metropolitan of Kermanshah.

\section{Materials and Methods}

The method of studying was descriptive-analytical as well as comparative. Given the geospatial nature of the present study; the Geographic Information System (GIS) was applied and additionally, the whole of data were inserted and analyzed in ArcGIS. The universe of the research consisted of gastrointestinal tract cancerstricken patients, whose information was documented in pathological centers of Kermanshah and their medical dossiers were collected by the health department of the medical school of Kermanshah. To identify and determine the temporal patterns of cancer in the City of Kermanshah, the statistical models of basic graphics and interpolation in the environment of GIS were utilized. The statistical tests of basic graphics include standard distance and mean center tests (equations $1 \& 2$ ).

$\begin{array}{ll}\text { Equation (1): } \mathrm{X}= & \sum_{i=1}^{N} \chi_{N_{i}} \sum_{i=1}^{N} Y_{i} \\ \text { Equation (2): SD: } & \sqrt{ }\left(\sum^{\mathrm{Di} 2} /{ }_{\mathrm{N}}\right)\end{array}$

The nearest neighbor index was applied to identify the clusters of the disease (equation 3):

Equation (3): NNI $=\quad \begin{aligned} & d(\mathrm{NN}) \\ & d(\mathrm{ran})\end{aligned}$

The Z-score is used to verify the nearest neighbor index. This test statistically determines how much the average distance from the nearest real neighbor is different from the average distance from the nearest random neighbor. The concept of Z-score test could be found in normal standard deviation tables. In principle, the more negative the $\mathrm{Z}$-score, the more reliable the results of the nearest neighbor index will be. In the classification of the spatial patterns into clustered, dispersed and random, the manner of the regional units' arrangement could be focused on. The similarity and dissimilarity of each pair of neighboring regional units could be measured. When such similarities and dissimilarities are summarized for the spatial patterns, the spatial autocorrelation is formed. There are diverse models for measuring the spatial autocorrelation statistics including Moran's I index and Getis-Ord general $\mathrm{G}$ being used in the present study (equations 4 \& 5).

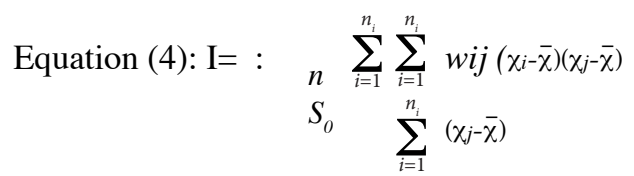

Equation (5): $\mathrm{G}(\mathrm{d})=\sum_{\substack{i=1 \\ n_{i}}}^{n_{i=1}} \sum_{i j}^{n_{i}} w_{i}{ }^{(d)} \chi_{i}-\chi_{j}$ $\sum_{i=1}^{n_{i}} \sum_{i=1}^{n_{i}}\left(\chi_{i}-\chi_{j=1}\right)$ 
The collected data were analyzed in terms of such variables as age, gender, the type of gastrointestinal tract cancer, the place where they are stricken by this cancer, the intensity pitch of the cancer and the behavior of the related tumor. In the present study, the intensity pitches of the cancer means the intensity of differentiations of the cancers which exist on the pathology sheets of patients and have been examined according to findings from tumor biopsy as well as the level of differentiation of the tissue. The intensity pitches of the cancer were classified based on the ICD-O coding system (1=good differentiation, $2=$ average differentiation, $3=$ weak differentiation, $4=$ without differentiation and $9=$ unknown differentiation). In addition to the above-mentioned statistical tests, the statistical method of interpolation of Kernel Density Test was applied to identify the loci of the disease. Similarly, based on the codes of ICD-O, the behavior of the tumor was classified into six levels of $0=$ benign, $1=$ unknown in terms of being malignant or benign, $2=$ carcinoma, $3=$ malignant disease of primary origin, $6=$ metastatic malignant disease and $9=$ malignant disease of unknown primary origin.23 There was a limitation in the present study. refer to sketchy and lost addresses that made the research team exclude them. This case might have certain meanings in the proper estimation of cancer incidence.

\section{Results}

\section{Descriptive statistics of the gastrointestinal tract cancer}

According to table (1), out of 283 cases of cancers within the space of 5 years, 157 and 126 cases were males and females, respectively. Stomach and colon cancers (each with $34.6 \%$ of incidence pitch) held the highest percentages compared to other cancers. In terms of age means, the anus cancer-stricken patients had the highest means and the small intestine cancer-stricken patients had the lowest means. Cancer has held an ascending trend over 2007-2012 and the pathological reports indicate that the number of cancer cases has increased from 23 cases in 2007 to 65 cases in 2012 (diagram 1).

\section{The standard distance and mean center tests}

The mean center of cancer had a trivial difference during the period under study (2007-2012) in the City of Kermanshah. So, the mean center of cancer has greatly overlaid the geographical center of the City of Kermanshah in 2007, 2008,2009, 2010 and 2011 and the mean center of cancer has gravitated towards the eastern part of the City of Kermanshah in 2012. The standard distance of cancer within the space of 5 years has had a northeast-southwest direction which indicates that the distribution of this disease is uniform across the City of Kermanshah. Not to mention, the distribution of this disease was gravitated towards the city center in 2007 and such a distribution extended towards the eastern parts of the City (figure 1).

\section{Clustering test (the nearest neighbor index)}

Clustering test is the first step for identifying the spatial patterns of the loci of diseases and the bigger the negative $\mathrm{Z}$-score, the more reliable the nearest neighbor index will be. According to figure (2), the value of Z-score for cancer is 18.08 , with a $\mathrm{P}=0.000$, which indicates that this disease holds a clustered feature in the City of Kermanshah.

\section{Clustering test (the Moran's I \& Getis-Ord general G indexes)}

The results of analyzing the Moran's I and Getis-Ord general G indexes related to cancer across the City of Kermanshah have been summarized and displayed in table (2). The Moran's I coefficient was positive and was equal to 0.05 which indicated that the spatial distribution of this disease held a clustered feature in the City of Kermanshah. Since, the more the Moran's I coefficient gravitates towards number 1 , the more concentrated the cluster will be . Considering the fact that $\mathrm{Z}$-score $=1.20$ and the expected value $=-0.010417$ (Z-score $>$ the expected value) at the confidence level of Sig .L $=0.01$, both the clustering feature of the disease distribution and the Moran's I coefficient were verified.

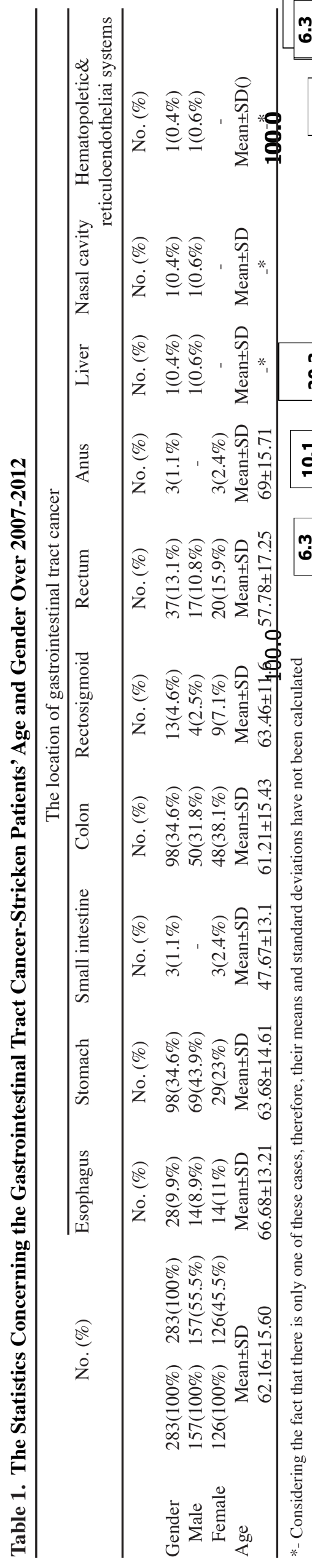




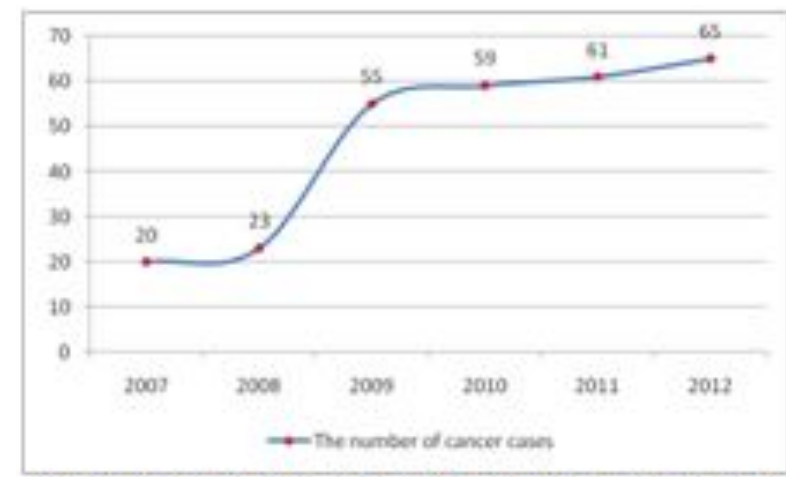

Diagram 1. The Frequency of Gastrointestinal Tract Cancer Over 2007-2012

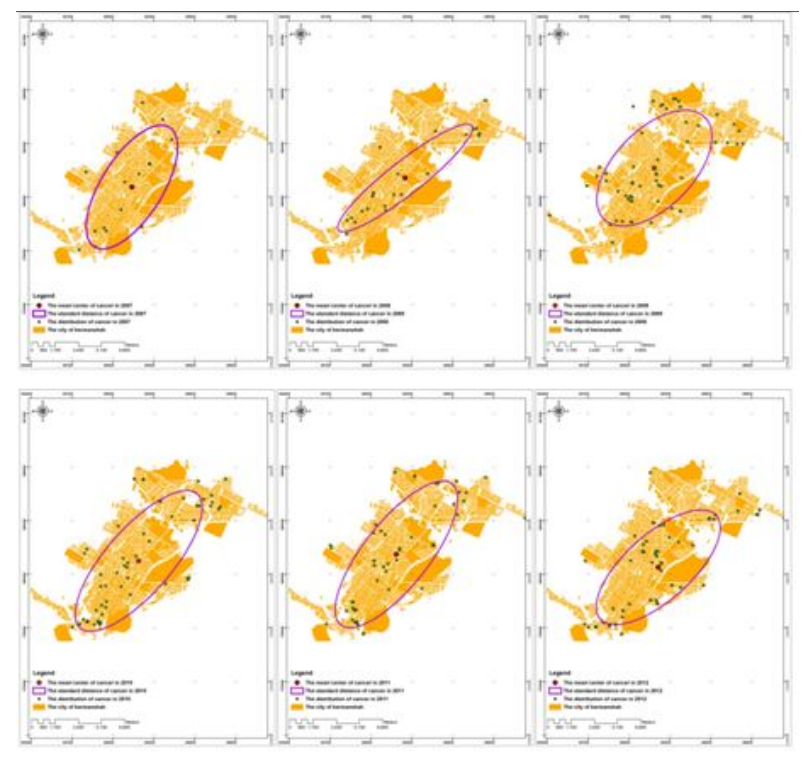

Figure 1. The Standard Distance and mean Center Tests of Cancer Over 2007-2012

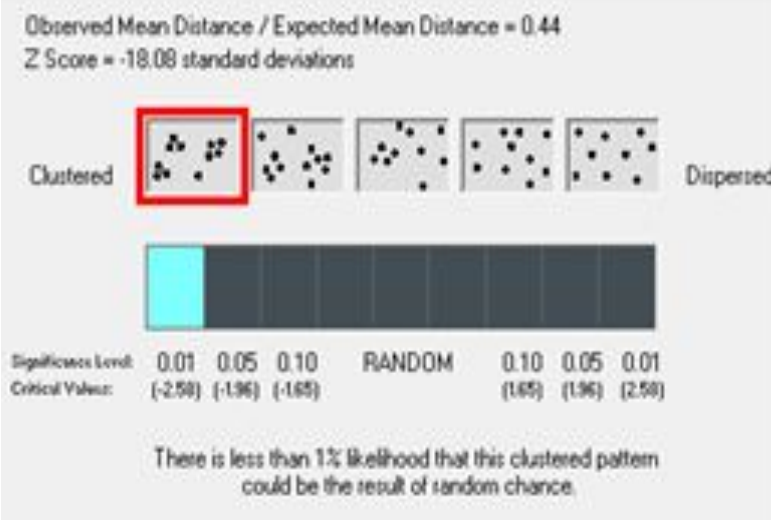

Figure 2. The Graphic Display of the Neareat Neighbor Index of Cancer in the Environment of GIS
The Moran's I index is well suited for statistical attributes and description of spatial correlations. But, this index is not efficient enough to identify the various types of spatial pattern classifications. These patterns are sometimes called "hot spots" and "cold spots" of concentration. For example, if high values are near each other, the Moran's I index and Gary ratio indicate a relatively high positive spatial autocorrelation and this cluster of high values may be called "hot spots". But, the high positive spatial autocorrelation shown by the Moran's I index and Gary ratio might have been created by the adjacent low values and this cluster of low values could be called "cold spots". The Moran's I index could not differentiate between these two types of spatial autocorrelations. The attained results showed that $\mathrm{G}=$ 0.000 and $Z$-score $=-1.11$ and indicated the spatial pattern of caner concentration across the City of Kermanshah was a type of low-high clusters (table 2).

Table 2. The Summary of the Results of Analyzing the Moran's I and Getis-Ord General G Indexes Related to Cancer Across the City of Kermanshah

\begin{tabular}{lccc}
\hline & The expected value & Variance & Z-score \\
\hline $\begin{array}{l}\text { Moran's I } \\
0.050395\end{array}$ & -0.01 & 0.002 & 1.2 \\
$\begin{array}{l}\text { Getis-Ord general G } \\
0.000065\end{array}$ & 0.00008 & 0 & -1.1 \\
\hline
\end{tabular}

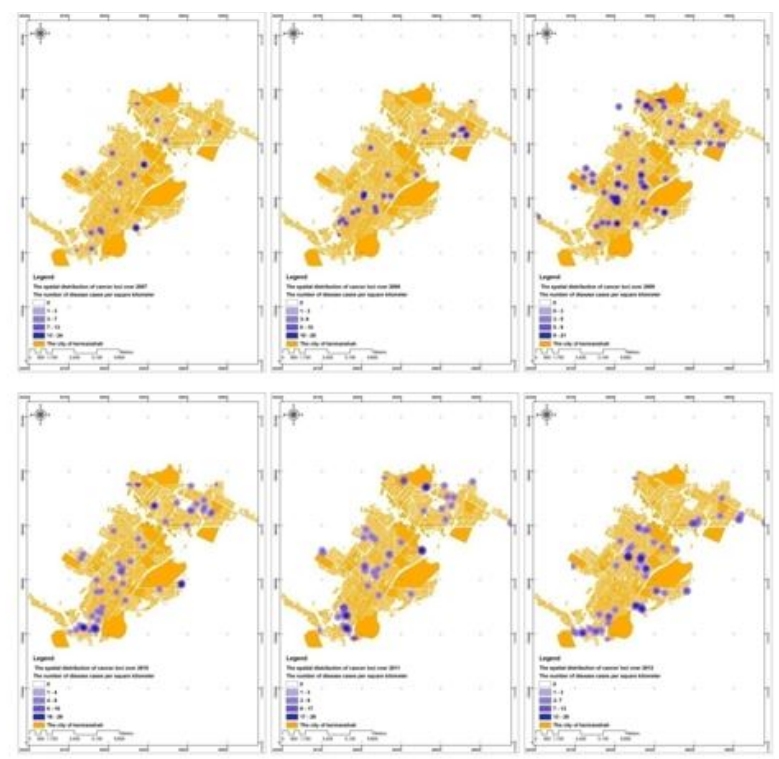

Figure 3. The Expensive Trend of Cancer within the Space of 5 years

Table 3. The Scope of the Most Important Loci of Gastrointestinal Tract Cancer in the City of Kermanshah Over 2007-2012

\begin{tabular}{|c|c|c|}
\hline Row & Item & The scope of disease loci \\
\hline 1 & $\begin{array}{l}\text { Level one: } \\
29-59 \text { cases of disease per square kilometer }\end{array}$ & $\begin{array}{l}\text { Ferdosi, Kasra, Bahar, } 22 \text { Bahman, Hafezieh (Cha- } \\
\text { ghagolan) and Jafarabad }\end{array}$ \\
\hline 2 & $\begin{array}{l}\text { Level two: } \\
\text { 15-29 cases of disease per square kilometer }\end{array}$ & $\begin{array}{l}\text { Jandarmeri, Sadeghieh, Saadi, Ariashahr, Kianshahr, } \\
\text { Farhangian1, Shaterabad, Gozarnameh, Farhang- } \\
\text { ian2, Chaghakabud, Hekmatabad, Baghabrisham, } \\
\text { Sorkhalijeh, Milad and Jahad. }\end{array}$ \\
\hline
\end{tabular}




\section{Kernel density test}

According to Kernel Density Test of the status of spatial distribution of cancer across the City of Kermanshah, the results of the prior tests were verified in another way and indicated that the distribution of gastrointestinal tract cancer within the scope under study was clustered, i.e. the cancer distribution was more concentrated in some parts of the City of Kermanshah (figure $3 \&$ table 3 ).

\section{Discussion}

The present research aimed to study the spatiotemporal distribution of gastrointestinal tract cancer through GIS over 2007-2012 in Kermanshah-Iran. This manuscript was the first report on cancer incidence in the metropolis of Kermanshah.

Enough studies have not been carried out regarding the maps of gastrointestinal tract cancer in different parts of Iran, especially in western provinces. So, there was no other choice left for the present study but to use the comparisons based on the epidemiological dimensions of gastrointestinal tract cancer.

According to the pathological reports of our study in the City of Kermanshah within the space of 5 years, 283 cases of gastrointestinal tract cancer were reported from which the highest percentage was related to males $(55.5 \%)$. This result concurred with the results of studies performed by Hajian et al (2003)(Haghighi and Hoseinni, 2003). This difference between men and women could be related to this issue, that they are more in contact with carcinogens, chemicals, pesticide, and x-ray radiation, and additionally, they consumed more alcoholic drinks, cigarette and drugs than women do. The mean age of our cases in overall was $62.16 \pm 15.60$ years old, which was similar to the results of studies performed in Mazandaran, Ardabil and Kurdistan and also east Azerbaijan (Esmaielee, 1992; Moulaie et al., 2000; Yazdanbod et al., 2005; Zeinalzadeh et al., 2012). The Results of our study showed that the incidence pitch of gastrointestinal tract cancer has increased, might be due to the improvement of registration manner of cancer data, the improvement of existing facilities to diagnose illnesses, aging as shown and illustrated in other similar studies(Darvishmoghadam et al., 2005).

In our study the mean center of cancer had a trivial difference during the period under study (2007-2012), So that, the mean center of cancer has greatly overlaid the geographical center of the City of Kermanshah in 2007, 2009 and 2011 and the mean center of cancer has gravitated towards the eastern part of the City of Kermanshah in the years of 2008 and 2012.

In addition, the standard distance of cancer within the space of 5 years has had a northeast-southwest direction. Not to mention, the distribution of this disease gravitated towards the city center, east and to some extent towards southeast in 2007-2008 and such a distribution extended towards the northern and northeastern neighborhoods of the City in 2009. Additionally, this disease was scattered across the whole of the City of Kermanshah in 20102011 and once again, it extended towards the eastern and southeastern parts of Kermanshah in 2012 which indicated a significant variation in terms of different years and regions. It seems that the variation in cancer distribution is under the influence of the expansion of city to the east, the type of cancer (in terms of the location of cancer) and the socio-economic bases of citizens. Given the fact that no corresponding research was found, there was not the possibility of comparison with other studies.

The results of the clustered tests in GIS environment demonstrated that the nearest neighbor index was 0.44 , $\mathrm{Z}=-18.08$ and $\mathrm{P}$-value $=0.00$ which indicated that the gastrointestinal tract cancer was clustered in the City of Kermanshah. The Moran's coefficient was 0.05 and Z-score was 1.20 which indicated that the disease distribution was clustered and also the Moran's coefficient was verified. In addition, Getis-Ord general $\mathrm{G}=0.000$ and Z-score $=-1.11$ which indicated that the spatial pattern of the cancer concentration was a type of low-high clusters. These results demonstrated that the gastrointestinal tract cancer followed a concentrated and clustered pattern in the City of Kermanshah,i.e. some loci of this disease have emerged in the City of Kermanshah and the Kernel Density Test was applied for identifying the temporal location of such loci of disease. The results of this test showed that the distribution of cancer was more concentrated in some parts of the City of Kermanshah, including 6 neighborhoods with 29-59 cases of disease per square kilometer and 15 neighborhoods with 15-29 cases of disease per square kilometer. This test confirmed the results of the previous tests to some extent. Some neighborhoods were high-class and some others were deprived, so there was the possibility that this type of distribution of the disease be under the influence of the cancers related to the upper and lower parts of gastrointestinal tract which required separate studies in this regard. In relation to the geographic map of cancer by GIS, the present study was similar to the study performed by Asmarian et al (2012) and RohaniRasaf et al (2011)(Rohani et al., 2011; Asmarian et al., 2013). The present study was different from the study undertaken by Asmarian et al (2012) in terms of the location scope of the research, i.e. the present study was a local one. Also, the present study was different from the study performed by RohaniRasaf et al (2011) in terms of precision to identify the loci of disease, i.e. the present study examined the spatiotemporal distribution of gastrointestinal tract cancer per square kilometer. Such clusters of cancers have been shown in other studies(Openshaw et al., 1987; Openshaw et al., 1988; Rohani et al., 2011).

In our research the gastrointestinal tract cancer has held an ascending trend within the time period of study (Diagram 1) and the spatiotemporal distribution of cancer followed a concentrated and clustered pattern in the City of Kermanshah, i.e. some loci of this disease have emerged in the City of Kermanshah. The geographic information system (GIS) is a practical and useful in creating awareness and identification of areas for intervention. Evaluation of risk factors is important and necessary for researchers. Therefore, awareness of the geographical distribution of cancer could provide detailed and accessible information for implementation of preventive activities. There is the need for more studies respecting the effectiveness of GIS not only for determining the etiology of cancers but also for helping policy makers to promote health care and preventive strategies. 


\section{Acknowledgements}

The kindly supports and assistance of our respectful colleagues in the Medical School of Kermanshah as well as the financial and spiritual supports of the deputy head of research \& technology department of the Medical School of Kermanshah are sincerely appreciated.

\section{References}

Askarian M, Goli A, Oroei M, et al (2014). The geographical clusters of gastrointestinal tract cancer in fars province, Southern Iran. International journal of preventive medicine, $\mathbf{5}, 857$.

Asmarian NS, Kavousi A, Salehi M (2013). Mapping of large intestine cancer incidence rate using area-to-area Poisson Kriging in Iran from 2003 to 2007. Razi Journal of Medical Sciences, 20, 10-7.

Auchincloss AH, Gebreab SY, Mair C, et al (2012). A review of spatial methods in epidemiology, 2000-2010. Annual review of public health, 33, 107.

Clarke KC, McLafferty SL, Tempalski BJ (1996). On epidemiology and geographic information systems: a review and discussion of future directions. Emerging infectious diseases, 2, 85.

Clayton D, Kaldor J (1987). Empirical Bayes estimates of age-standardized relative risks for use in disease mapping. Biometrics, 671-81.

Curtis AB, Kothari C, Paul R, et al (2013). Using GIS and secondary data to target diabetes-related public health efforts. Public Health Reports, 128, 212.

Darvishmoghadam S, Abbasi MH, Zeinalinejad H (2005). The incidence rate of gastrointestinal tract cancers in Kerman province during 1996-2000. Journal of Kerman University of Medical Sciences, 12, 153-8.

Esmaielee H (1992). Comparing survival rate and its characteristics of esophagus and stomach cancer in Mazandran province. School of Medicine Science, Tarbiat Modarres Univ, 18-39.

Fathollahi MS, Mahmoodi M, Mohammad K, et al (2011). Expected survival using models of life table compared with survival of gastrointestinal tract cancer patients in north of iran. Iranian journal of public health, 40, 74 .

Haghighi F, Hoseinni M (2003). Attitude and performance Assessment of Birjand population to cancer and screening of that. Birjand University of Medical Sciences, 10, 15-8.

Hajmanoochehri F, Asefzadeh S, Kazemifar AM, et al (2014). Clinicopathological features of colon adenocarcinoma in Qazvin, Iran: a 16 year study. Asian Pac J Cancer Prev, 15, 951-5.

Krieger N (2000). Epidemiology and social sciences: towards a critical reengagement in the 21 st century. Epidemiologic Reviews, 22, 155-63.

Mahbubeh S, Holakouie-Naieni K, Mostafavi E, et al (2014). Spatial Analysis of Multiple Sclerosis Disease in Tehran Metro-poliา $\tan$ Zone, Iran, 2001-2012. Iranian journal of public health, 43, 621-9.

May JM (1950). Medical geography: its methods and objectives. Geographical Review, 9-41.

McLeod KS (2000). Our sense of Snow: the myth of John Snow in medical geography. Social science \& medicine, 50, 923-35.

Mehrabani D, Tabei S, Heydari S, et al (2008). Cancer occurrence in Fars Province, Southern Iran. Iranian Red Crescent Medical Journal, 2008, 314-22.

Merletti F, Galassi C, Spadea T (2011). The socioeconomic determinants of cancer. Environ Health, 10, 7.

Mohammadpour Tahamtan RA, Yazdani J, Zare S, et al (2014). Geographical mapping of gastrointestinal cancer mortality rates in Mazandaran and Golestan provinces, Iran. Journal of Mazandaran University of Medical Sciences (JMUMS), 23.
Morra P, Bagli S, Spadoni G (2006). The analysis of human health risk with a detailed procedure operating in a GIS environment. Environment International, 32, 444-54.

MOULAEI N, Yazdanpanah K, Reshadmanesh N, et al (2000). Epidemiology of Gastric and Oesophagial Cancers In Kurdistan Province In 1999.

Murphy G, Pfeiffer R, Camargo MC, et al (2009). Meta-analysis shows that prevalence of Epstein-Barr virus-positive gastric cancer differs based on sex and anatomic location. Gastroenterology, 137, 824-33.

Musa GJ, Chiang P-H, Sylk T, et al (2013). Use of GIS Mapping as a public health tool - from cholera to cancer. Health Services Insights, 6, 111 .

Openshaw S, Charlton M, Craft AW, et al (1988). Investigation of leukaemia clusters by use of a geographical analysis machine. The Lancet, 331, 272-3.

Openshaw S, Charlton M, Wymer C, et al (1987). A mark 1 geographical analysis machine for the automated analysis of point data sets. International Journal of Geographical Information System, 1, 335-58.

Pearce J, Boyle P (2005). Examining the relationship between lung cancer and radon in small areas across Scotland. Health \& place, 11, 275-82.

Radmard AR (2010). Five common cancers in Iran. Archives of Iranian Med, 13, 143-6.

RAHIMI F, HEIDARI M (2012). Time trend analysis of stomach cancer incidence in the west of Iran.

Rezaeian M (2004). An introduction to the practical methods for mapping the geographical morbidity and mortality rates. Tollo-e-behdasht, 2, 41-51.

Roche LM, Skinner R, Weinstein RB (2002). Use of a geographic information system to identify and characterize areas with high proportions of distant stage breast cancer. J Public Health Management and Practice, 8, 26-32.

ROHANI RM, ROHANI RM, Rahimi F, et al (2011). Distribution of cancer incidence in districts and neighbourhoods of a number of Tehran districts in 1386.

Sadjadi A, Malekzadeh R, Derakhshan MH, et al (2003). Cancer occurrence in Ardabil: Results of a population based Cancer Registry from Iran. International J Cancer, 107, 113-8.

Samat N, Shattar AKA (2014). Spatial accessibility of colorectal cancer patients to health care facilities in kelantan state: a study on medical geography. World Applied Sciences Journal, 31, 1772-82.

Shad R, Ebadi H, Ghods M (2003). Evaluation of Route Finding Methods in GIS Application. Tehran: Department of Geodesy and Geomatics Enginering, Khajeh Nasi Toosi University of Technology; . Received: 21.11.2010 Accepted: 25.6. Journal of Isfahan Medical School Original Article, 29.

Snow J 1855. On the mode of communication of cholera, John Churchill.

Somi MH, Mirinezhad K, Farhang S, et al (2006). Gastrointestinal cancer occurrence in East Azarbaijan: a five year study from North Western Iran. Asian Pac J Cancer Prev, 7, 309.

Wu K-S, Huo X, Zhu G-H (2008). Relationships between esophageal cancer and spatial environment factors by using Geographic Information System. Science of the total environment, 393, 219-25.

Yazdanbod E, Samadi F, Malekzade R, et al (2005). Four-Year Survival Rate of Patients with Upper GI Cancer in Ardabil. Journal of Ardabil University of Medical Sciences, 5, 180-4.

Zeinalzadeh AH, Kousha A, Abdullahi L, et al (2012). Pattern of age distribution of different cancers in East Azerbaijan province, Iran. Journal of Kerman University of Medical Sciences, 19, 308-86.

Zulu LC, Kalipeni E, Johannes E (2014). Analyzing spatial clustering and the spatiotemporal nature and trends of HIV/ AIDS prevalence using GIS: the case of Malawi, 1994-2010. BMC Infectious Diseases, 14, 285. 